\title{
Existing population projections in Sri Lanka: comparative analysis of the accuracy
}

\author{
Ranjith de Silva ${ }^{1^{*}}$ and W. Indralal De Silva ${ }^{2}$ \\ ${ }^{1}$ Sri Lanka College of Obstetricians and Gynaecologists, No: 112, Model Farm Road, Colombo 08, Sri Lanka. \\ ${ }^{2}$ University of Colombo, Colombo 03, Sri Lanka.
}

\begin{abstract}
The aim of the study is to evaluate the accuracy of past population projections with the comparison of observed figures in 2001 and 2012 population censuses in Sri Lanka. It has also compared the age-sex structures of the projections with the observed data. Out of the six population projections, four had been produced by three local forecasters: Soma De Silva, W. Indralal De Silva, A. T. P. L. Abeykoon and the other two by United Nations and, all of these projections had been examined. Standard variation of four projections produced before year 2000 show a percentage error ranging from 1.16 to 3.05 with 2001 observed figures and 3.33 to 5.21 with 2012 observed figures. Other two projections produced after year 2000 have a percentage error 1.59 and -3.09 , respectively, when compared with 2012 observed figures. Accuracy of observed vs. projected population, projection errors and their measurement as well as age-sex structures of two projections against 2012 observed population have been examined. The examination of accuracy of the past projection results would lead to a generation of more reliable projections in future.
\end{abstract}

Keywords: Population projection, accuracy, errors, deviation, forecast.

\section{INTRODUCTION}

Population projections are frequently used by policy makers for policy formulation, programme implementation and monitoring. National and subnational level population projections are used by policy makers, planners and demographers at local, regional and national levels to project future demand for human necessities and to forecast future demographic characteristics. Generally, it becomes a part of policy formulation and, additionally, it is usable for decisionmaking processes. Thus, it is very important to produce more accurate projections of population for policy formulation and implementation.

The population projections are based on the envisaged future changes of three components of the population: fertility, mortality and migration. Expected future changes in these components need to be assumed in a justifiable manner by considering several socioeconomic and cultural phenomena of the population. However, making appropriate assumptions seem to be a very difficult task to produce highly accurate population projections. Expected accuracy of the projected results does not include in the procedure of population projection itself. Nevertheless, the accuracy is an important aspect of the quality of the projection.

The aim of this study is to evaluate the accuracy of past population projections by comparison of those forecasts with actually observed figures of population censuses in 2001 and 2012 in Sri Lanka. It would also compare age-sex structures of the projections with the observed structure. The comparison of the assumptions for fertility, mortality and migration schedules made by the forecasters for the projected periods has also been considered. Six population projections have been included in this evaluation. Four of them were produced by three local forecasters, Soma De Silva, W. Indralal De Silva, A. T. P. L. Abeykoon and the other two were United Nations (UN) population projections for

"Corresponding author (ranjith.desilva49@gmail.com) 
Sri Lanka. The evaluation of accuracy of the results of past projections would definitely lead to a generation of more reliable projections in future.

\section{Studies on accuracy: observed vs. projected population}

Studies conducted to evaluate the accuracy of population projections in Sri Lanka are hardly available. However, Norbert (1993) discussed the population growth in Sri Lanka reviewing different projection models. Selvaratnam (1959) and Selvaratnam et al. (1970) developed population projections for Ceylon. In these 3 studies researchers have not attempted to evaluate the accuracies of population projections. The closest reason for the unavailability of such studies could be the less number of projections carried out by local forecasters. Khan \& Lutz (2007) evaluate past UN population projections and examine how to anticipate demographic trends in six Southeast Asian countries. They have clearly noted that in many cases, the base line error (error in base population) was even more significant than the change error (errors in assumptions on fertility, mortality and migration) for the accuracy of projection figures. One important point which they have expressed is that country specific culture and politics do matter to a great extent for the specific paths of fertility and mortality. It is a fact that, there is no theory available to evaluate how specific cultural, social, economic and political conditions would effect fertility, mortality and migration. They have also mentioned that almost all the projections would also have some errors in general.

Keilman (1998) discusses in his study on the accuracy of the United Nations World Population Projections. He has highlighted considerable differences of the accuracy of UN projections on aggregate level of seven major regions (Africa, Asia, Europe, the former Soviet Union, North America, Latin America and Oceania). He has found that difference in accuracy is also seen in a few large countries such as India, China, and the United States. One of the interesting findings of Keilman's study is that the assumptions of future mortality for Africa and Asia seem to be relatively difficult to accept. On the other hand, errors in fertility schedules used for Asia, North America and Latin America are above the world average.

A research article on "Forecast Accuracy and Uncertainty of Australian Bureau of Statistics, State and Territory Population Projections" by Wilson (2012) tends to determine: how well Australian Bureau of Statistics (ABS) pertaining to state and territory projections perform as forecasts of future population, how accurate were projections of total population and age-specific populations. Among several findings of this study, below are important: "There was an approximately linear relationship between average error and projection duration and a similar relationship between the $80 \%$ distribution of errors and projection duration", "Errors in net inter-state migration and net overseas migration projections were the major contributions to total population forecast error", "Projections of age-specific populations were generally less accurate for $0-4$ years old than older children, often quite inaccurate at the peak migration ages, and in some cases quite inaccurate at the very oldest ages" (Wilson, 2012).

Stoto (1983) states that although population projections are used for many planning and policy studies, the projections are inherently inaccurate. His study evaluates past population projection errors and constructs confidence intervals for future projections for the United States in the year 2000. Confidence intervals can be computed only for the error in total population size, and not the error in age composition.

A study of Indonesian population projection 20102035 by McDonald (2014) concludes that a small variation in fertility impacts substantially on population growth in the youngest age group. Comparing the official Indonesian government projections and the UN projections, it has been found that the assumptions on current and future fertility made by both the projections are roughly similar but initial fertility is always lower in UN projections. In terms of improvement of life expectancy, UN projections assume 3.7 years while the official projection assumes 2.1 years for the period from 2010-14 to 2030-34. Although the base population for official projection is lower than the base population used by UN in 2010 by 2.2 million, all the UN projected population figures in 2020, 2030 and 2035 are lower than the official projection by 1.7, 2.9 and 2.3 million, respectively.

\section{Projection errors and their measurement}

Different types of errors are identified in population projections. Basically, these errors can be divided into two main categories: baseline errors or estimating of the starting conditions and trend errors or on assumptions of future trends. On the other hand, errors can be classified according to different types of sources. Kahn \& Lutz (2007) classify sources of errors with reference to Keilman (1998) as follows.

(a) Errors in projection input data: the base year population, incorrect age-sex structure due to misreporting or underreporting of censuses or incorrect estimates from the observed figures. 
(b) Incorrect specifications of forecast parameters: assumptions made in fertility, mortality and migration for the projected period.

(c) Unexpected events: such as outbreak of war, impact of epidemics, natural disasters such as tsunami, floods, cyclone and famine and the urgency of skill migration.

(d) Randomness in parameters: stochastic fluctuations in the estimated number of births, deaths and migrations not taking into account in projection method.

(e) Inaccurate projection model specifications: possibility of omitting one of the three components of fertility, mortality and migration from the projection model.

Sometimes these errors work in different directions and partly compensate each other. The possibility of impact of errors in the same direction are reinforcing each other (Kahn \& Lutz, 2007).

Several indicators have been developed to measure the errors in past projections. The simplest and the most common indicator that has been developed is measuring the Percentage Error (PE) in terms of deviation from the observed figure. This is a relative measure which summarises the results of the comparison between two different projections. $\mathrm{PE}$ is defined as;

$$
\mathrm{PE}=\left(\frac{\text { projected }- \text { observed }}{\text { observed }}\right) \times 100
$$

Three scenarios can be explained based on the value of "PE".

If $\mathrm{PE}=0$, the projection is perfect with no errors.

If $\mathrm{PE}$ has a positive value, the projection is high with an overestimation.

If $\mathrm{PE}$ has a negative value, the projection is low with an underestimation.

Absolute Percentage Error (APE) is computed by taking out the sign of the PE.

To evaluate a common error in a series of forecasts, the mean percentage error (MPE) provides an average measure of bias: a positive MPE indicates that forecasts tended to be too high on average, and a negative MPE reflects forecasts that were too low. Similarly, Mean Absolute Percentage Error (MAPE) is calculated by taking out the sign of the MPE.

\section{SOURCES OF DATA AND METHODOLOGY}

Six projections which were published after year 1990 have been taken to compare with observed population figures of 2001 and 2012 censuses. Details of projections are given in the Table 1 .

Table 1: Details of population projections available for Sri Lanka since 1990s

\begin{tabular}{|c|c|c|c|c|c|}
\hline No. & Publication & Publisher & Author & $\begin{array}{r}\text { Date of } \\
\text { publication }\end{array}$ & $\begin{array}{r}\text { Projection } \\
\text { Period } \\
\end{array}$ \\
\hline 1 & $\begin{array}{l}\text { Population and Labour Force } \\
\text { Projections for Sri Lanka 1991-2031 }\end{array}$ & $\begin{array}{l}\text { Department of Census and } \\
\text { Statistics, Sri Lanka }\end{array}$ & Soma De Silva & March 1994 & $1991-2031$ \\
\hline 2 & $\begin{array}{l}\text { Population Projections for Sri Lanka: } \\
\text { 1991-2041 }\end{array}$ & $\begin{array}{l}\text { Institute of Policy Studies, } \\
\text { Sri Lanka }\end{array}$ & W. Indralal De Silva & August 1997 & 1991-2041 \\
\hline 3 & $\begin{array}{l}\text { Population and Manpower Resources } \\
\text { of Sri Lanka }\end{array}$ & $\begin{array}{l}\text { Natural Resources, Energy and } \\
\text { Science Authority of Sri Lanka, } \\
\text { Sri Lanka }\end{array}$ & A. T. P. L. Abeykoon & 1998 & $1995-2030$ \\
\hline 4 & $\begin{array}{l}\text { World Population Prospects: } \\
\text { The } 1996 \text { Revision }\end{array}$ & United Nations, New York & $\begin{array}{l}\text { Department of Economics } \\
\text { and Social Affairs } \\
\text { Population Division }\end{array}$ & 1998 & $1995-2050$ \\
\hline 5 & $\begin{array}{l}\text { A Population Projection of Sri Lanka } \\
\text { For The New Millennium 2001-2101: } \\
\text { Trends and Implications }\end{array}$ & $\begin{array}{l}\text { Institute for Health Policy, } \\
\text { Sri Lanka }\end{array}$ & W. Indralal De Silva & June 2007 & $2001-2101$ \\
\hline 6 & $\begin{array}{l}\text { World Population Prospects } \\
\text { The } 2006 \text { Revision } \\
\text { Volume II: Sex and Age Distribution of } \\
\text { the World Population }\end{array}$ & United Nations, New York & $\begin{array}{l}\text { Department of Economics } \\
\text { and Social Affairs } \\
\text { Population Division }\end{array}$ & 2007 & $2005-2050$ \\
\hline
\end{tabular}


During the period of 1994-2007, four population projections have been published by local forecasters. Of the four, two have been prepared by W. Indralal De Silva; one in August 1997 and the latest other in June 2007. The oldest projection by Soma De Silva was published in March 1994. In 1998, A. T. P. L. Abeykoon has published his projection results. Two UN population projections which were published in 1998 and 2007 have been selected for comparison purposes.

All six projections are divided into two groups based on the year of publication being before and after year 2000, for comparison and evaluation of accuracy. First group consists of 4 projections viz. Soma De Silva (1994), W. Indralal De Silva (1997), A. T. P. L. Abeykoon (1998) and United Nations (1998). These four projections are ready for comparison among them and also to check for the accuracy against the observed results of censuses in 2001 and 2012.
The second group has only two projections, W. Indralal De Silva (2007) and United Nations (2007) which are eligible to use for checking the accuracy with latest 2012 population census figures.

Table 2 gives basic parameters of selected six projections. Except for the projection made by W. Indralal De Silva (2007), time horizon of rest of the projections is ranging 40-55 years. W. Indralal De Silva (2007) has made a fairly long term projection covering a period of 100 years in time span.

The methodology adopted for comparison and evaluation of projections has following steps:

1. Comparison of base populations and differences;

2. Calculation of PEs for group one projections in terms of total population against 2001 and 2012 observed;

Table 2: Projection parameters of six projections

\begin{tabular}{lllcrr}
\hline Group & No. & Author & Base year & Variants & $\begin{array}{r}\text { Time } \\
\text { horizon (years) }\end{array}$ \\
\hline One & 1 & Soma De Silva (March 1994) & 1991 & Standard, High, Low & 40 \\
& 2 & W. Indralal De Silva (August 1997) & 1991 & Standard, High, Low & Single variant \\
& 3 & A. T. P. L. Abeykoon (1998) & 1995 & Medium, High, Low & 55 \\
& 4 & United Nations (1998) & 1995 & & \\
& & & 2001 & Standard, High, Low & 100 \\
Two & 5 & W. Indralal De Silva (June 2007) & 2005 & Medium, High, Low & 45 \\
\hline
\end{tabular}

Sources: De Silva, S. (1994); De Silva, W. I. (1997 \& 2007); UN (1998 \& 2007); Abeykoon (1998).

Table 3: Differences in base year populations of 1991 by Soma De Silva and W. Indralal De Silva

\begin{tabular}{|c|c|c|c|}
\hline Age group & Soma De Silva (March 1994) & W. Indralal De Silva (August 1997) & Difference \\
\hline Total population ('000) & $17,259.1$ & $17,015.0$ & 244.1 \\
\hline $0-14$ & $5,390.4$ & $5,337.0$ & 53.4 \\
\hline $15-59$ & $10,469.3$ & $10,285.0$ & 184.3 \\
\hline $60+$ & $1,399.4$ & $1,393.0$ & 6.4 \\
\hline Male population ('000) & $8,735.8$ & $8,505.0$ & 230.8 \\
\hline $0-14$ & $2,720.6$ & $2,689.0$ & 31.6 \\
\hline $15-59$ & $5,295.0$ & $5,101.0$ & 194.0 \\
\hline $60+$ & 720.2 & 715.0 & 5.2 \\
\hline Female population ('000) & $8,523.3$ & $8,510.0$ & 13.3 \\
\hline $0-14$ & $2,669.8$ & $2,648.0$ & 21.8 \\
\hline $15-59$ & $5,174.3$ & $5,184.0$ & -9.7 \\
\hline $60+$ & 679.2 & 678.0 & 1.2 \\
\hline
\end{tabular}

Sources: De Silva, S. (1994); De Silva, W. I. (1997). 
3. Calculation of PEs for group two projections in terms of total population against 2012 observed;

4. Comparison of projected age-sex structures in 2012 of group two projections with 2012 observed. This comparison cannot be performed with 2001 census data due to non-availability of age-sex structure for 2001.

\section{COMPARISON AND EVALUATION}

\section{Base Populations}

Among the four projections in group one, 1991 mid-year population has been used as the base population in first two projections and 1995 mid-year population has been used as base in the other two projections. Due to different base year populations of two projections, which are 2001 and 2005, comparison of base year populations of group two projections is not practical (Table 2). The methods of computation of base populations of first two projections in group one are described as follows.

Base population of 1991 in Soma De Silva's projection: Population is projected based on the 1981 census data after adjusting under enumerations and brought forward to the midpoint of the year 1981. And then it is projected to 1991 considering observed trends in vital rates for a good portion of the 1981-91 period (De Silva, 1994).

Base population of 1991 in W. Indralal De Silva's (1997) projection: the 1981 population is brought forward to 1986 and then from 1986 to 1991, by using the observed trends in fertility, mortality and international migration (End Note 1).

Although in both projections 1991 mid-year population has been used as a base, differences in total population, male \& female population and agesex structures of two base years are highlighted. The differences are shown in Table 3.

Figures produced for the base year population of 1991 are 17.3 and 17.0 million for Soma De Silva (1994) and W. Indralal De Silva (1997), respectively. W. Indralal De Silva produced a base population of 1991, which is about 250,000 persons lower than the first author Soma De Silva. Population figures of each broad age group are also lower in W. Indralal De Silva's (1997) projection than those of Soma De Silva, except female age group 15-59. It is noted that the difference of male age group 15-59 is significantly higher than all the other age groups while the same age group for female takes a negative value.

To compare base year populations of $3^{\text {rd }}$ and $4^{\text {th }}$ projections in group 1, the methods of computations of their base populations are given below.

The base population of 1995 in Abeykoon's projection: it is estimated based on the population agesex structure of the 1981 census of population and the trends in fertility, mortality and migration during 1981 to 1995. It was then adjusted on the basis of estimates made in the Demographic Survey 1994 (Abeykoon, 1998).

The base population of 1995 in UN (1998) projection: As reported by UN, "estimated to be consistent with the 1981 census, and with estimated subsequent trends in fertility, mortality and migration" (UN, 1998). Table 4 compares figures in both base populations and its differences in the above two projections.

Figures of total population and male population in Abeykoon's base year are higher than UN's while total

Table 4: Differences in base year populations by Abeykoon and United Nations

\begin{tabular}{|c|c|c|c|}
\hline Indicator & Abeykoon (1998) & United Nations (1998) & Difference \\
\hline Total population ('000) & $18,127.0$ & $17,928.0$ & 199.0 \\
\hline $0-14$ & $5,022.0$ & $5,324.6$ & -302.6 \\
\hline $15-59$ & $11,461.0$ & $11,025.7$ & 435.3 \\
\hline $60+$ & $1,644.0$ & $1,577.7$ & 66.3 \\
\hline Male population ('000) & $9,153.0$ & $8,911.0$ & 242.0 \\
\hline Female population ('000) & $8,974.0$ & $9,017.0$ & -43.0 \\
\hline
\end{tabular}

Sources: Abeykoon (1998); UN (1998). 
female population is lower. Significant differences can be seen in the total population figures of 0-14 and 15-59 age groups. For instance, the child population (aged 0-14 years) reported in the 1995 base year of the UN projection is 5.3 million, which is higher than the corresponding figure (5.0 million) reported by Abeykoon (1998).

\section{Percentage Error (PE) in group one projections against observed population of 2001 and 2012}

Except Abeykoon's projection, all other 3 projections prepared with three variants viz standard (medium), high and low. The standard or medium variant is the one made on the most justifiable assumptions and also users most often select as the best guess. Thus, PEs can be calculated per each variant of projections against 2001 and 2012 observed. Projected figures of total population (standard variant) in the 3 projections of group one and Abeykoon's projected figures are plotted in line graphs against the 2001 and 2012 observed total population which represent bars in Figure 1.

It is clearly indicated that deviations from 2012 observed are always higher than the deviations from 2001 observed for all the projections. This pattern clearly indicate that projected size of a population of a given population tends to be more accurate for shorter period from the base year.

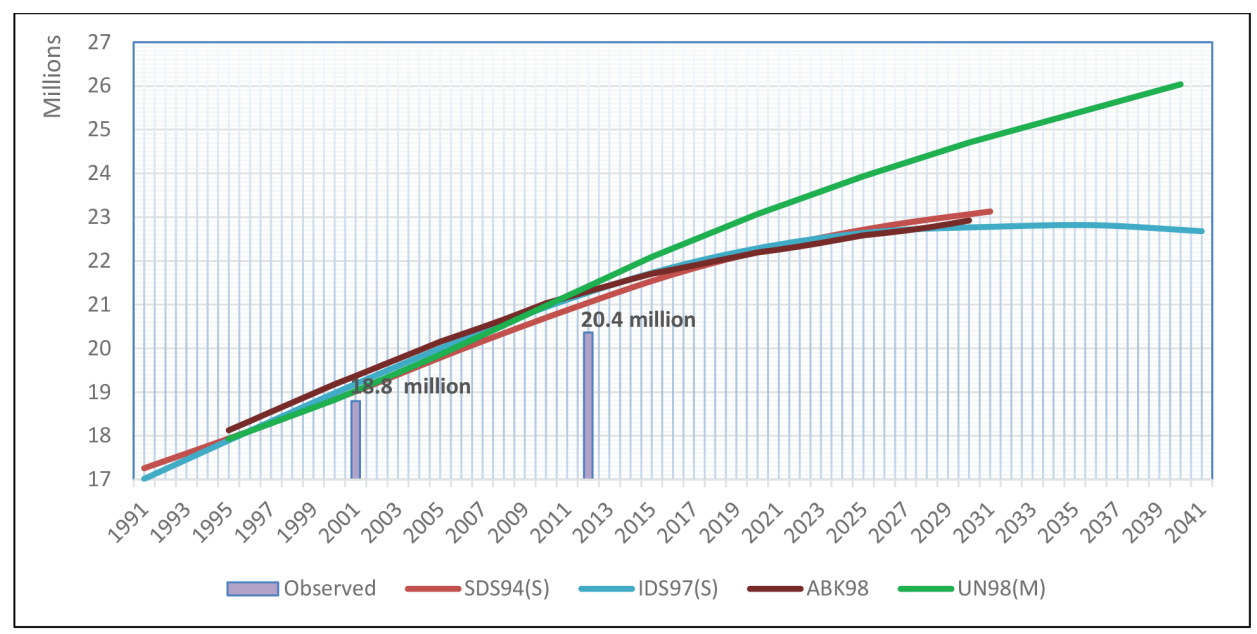

Figure 1: Comparison of projected (standard variant) population figures of $2001 \& 2012$ with observed figures

Note $\quad$ : SDS94(S) - Soma De Silva's 1994 standard variant, IDS97(S) - W. I. De Silva's 1997 standard variant, ABK98 - Abeykoon's 1998 projection, UN98(M) - United Nation's 1998 medium variant

Sources : De Silva, S. (1994); De Silva, W. I. (1997); UN (1998); Abeykoon (1998); Sri Lanka, Deptartment of Census and Statistics (2014)

Table 5: Percentage error (PE) derived by comparing projected population figures (standard variant) of 2001 and 2012 with observed figures

\begin{tabular}{|c|c|c|c|c|c|c|}
\hline \multirow[b]{2}{*}{ Projection } & \multicolumn{3}{|c|}{$\begin{array}{l}2001 \text { observed ('000) } \\
(18,797.3 \text { persons) }\end{array}$} & \multicolumn{3}{|c|}{$\begin{array}{c}2012 \text { observed ('000) } \\
(20,359.4 \text { persons) }\end{array}$} \\
\hline & $\begin{array}{r}\text { Projected } \\
\text { (Standard) } \\
\left({ }^{\prime} 000\right)\end{array}$ & Actual error & PE & $\begin{array}{r}\text { Projected } \\
\text { (Standard) } \\
\left({ }^{\circ} 000\right)\end{array}$ & Actual error & $\mathrm{PE}$ \\
\hline Soma De Silva (March 1994) & $19,015.3$ & 218.0 & 1.16 & $21,036.7^{*}$ & 677.3 & 3.33 \\
\hline $\begin{array}{l}\text { W. Indralal De Silva (August } \\
\text { 1997) }\end{array}$ & $19,185.8$ & 388.5 & 2.07 & $21,262.4^{*}$ & 903.0 & 4.44 \\
\hline A. T. P. L. Abeykoon (1998) & $19,370.0$ & 572.7 & 3.05 & $21,306.0$ & 946.6 & 4.65 \\
\hline United Nations (1998) & $19,028.4^{*}$ & 231.1 & 1.23 & $21,420.8^{*}$ & $1,061.4$ & 5.21 \\
\hline
\end{tabular}

Sources: De Silva, S. (1994); De Silva, W. I. (1997); UN (1998); Abeykoon (1998); Sri Lanka, Department of Census and Statistics (2014) 
The above graphical presentation has clearly shown that deviation of UN projection from others starting 2012 onwards. The calculated PEs for all four projected figures against $2001 \& 2012$ observed are shown in Table 5.

Due to higher figures of all four projections than observed in both 2001 and 2012, the sign of all PE values are positive. Soma De Silva's projection has reported minimum errors against both 2001 and 2012 observed. Out of all the four projections, UN projection reports second smallest PE against 2001 observed and the largest PE against 2012 observed. It is evident that
PEs on 2001 observed is always lower than PEs on 2012 observed. The mean absolute percentage error (MAPE) of four projections at 2001 and 2012 are 1.88 and 4.41 respectively. Thus, over the period of 2001-2012 the MAPE has increased by 2.53 per cent.

Figure 2 presents the comparison of high variant projection results of Soma De Silva (1994), W. Indralal De Silva (1997) and United Nations (1998) with the observed population of 2001 and 2012. The same comparison in the graphical presentation for low variant projections of Soma De Silva (1994), W. Indralal De Silva (1997) and United Nations (1998) is given in Figure 3.

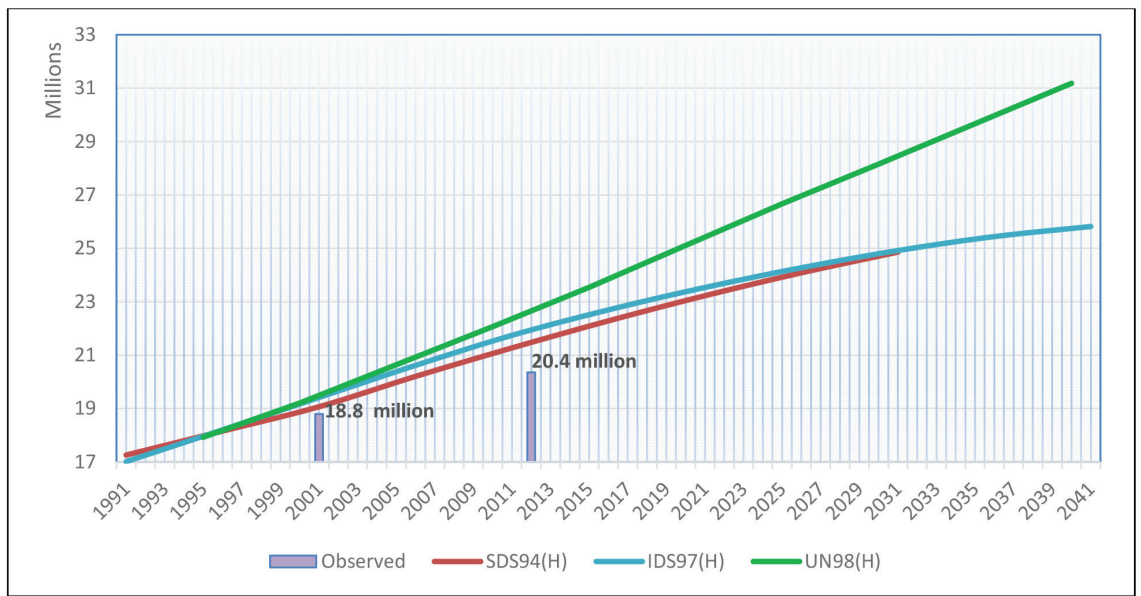

Figure 2: Comparison of projected (high variant) population figures of $2001 \& 2012$ with observed figures

Note : SDS94(H) - Soma De Silva's 1994 high variant, IDS97(H) - W. I. De Silva's 1997 high variant, UN98(H) - United Nation's 1998 high variant

Sources : De Silva, S. (1994); De Silva, W. I. (1997); UN (1998); Sri Lanka, Department of Census and Statistics (2014)

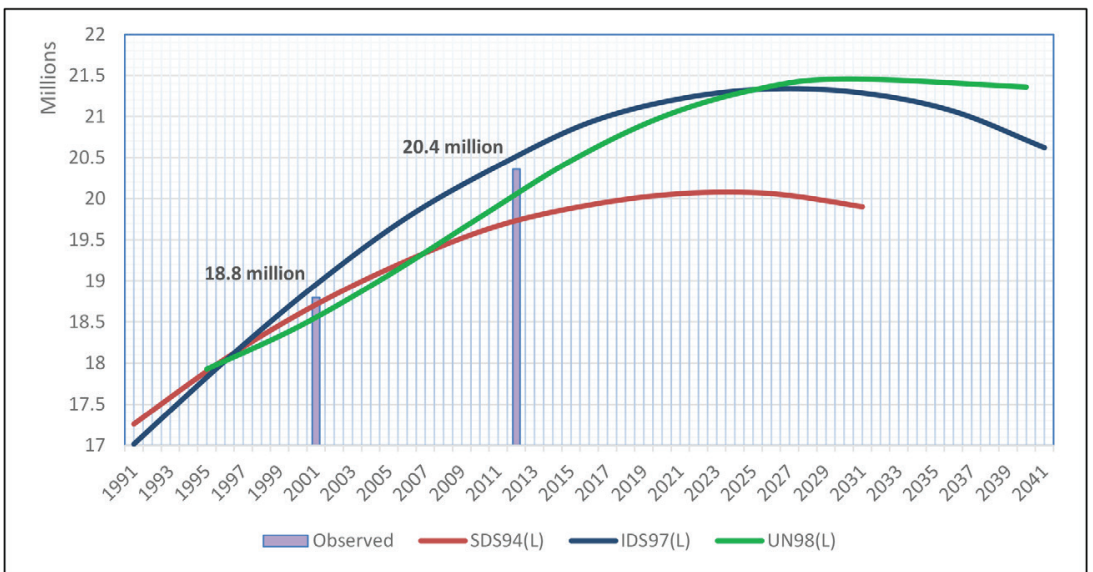

Figure 3: Comparison of projected (low variant) population figures of $2001 \& 2012$ with observed figures

Note : SDS94(L) - Soma De Silva’s 1994 low variant, IDS97(L) - W. I. De Silva’s 1997 low variant, UN98(L) - United Nation's 1998 low variant

Sources : De Silva, S. (1994); De Silva, W. I. (1997); UN (1998); Sri Lanka, Department of Census and Statistics (2014) 
Table 6: Percentage error (PE) derived by comparing projected population figures (high and low variants) of 2001 and 2012 with observed figures of group one

\begin{tabular}{|c|c|c|c|c|c|c|}
\hline \multirow[t]{2}{*}{ Projection } & \multicolumn{3}{|c|}{$\begin{array}{c}2001 \text { observed ('000) } \\
(18,797.3 \text { persons) }\end{array}$} & \multicolumn{3}{|c|}{$\begin{array}{c}2012 \text { observed ('000) } \\
(20,359.4 \text { persons) }\end{array}$} \\
\hline & Projected & Actual error & $\mathrm{PE}$ & Projected & Actual error & $\mathrm{PE}$ \\
\hline \multicolumn{7}{|l|}{ High variant } \\
\hline Soma De Silva (March 1994) & $19,068.5$ & 271.2 & 1.44 & $21,474.4^{*}$ & $1,115.0$ & 5.48 \\
\hline W. Indralal De Silva (August 1997) & $19,415.3$ & 618.0 & 3.29 & $21,933.3^{*}$ & $1,573.9$ & 7.73 \\
\hline United Nations (1998) & $19,496.8^{*}$ & 699.5 & 3.72 & $22,657.8^{*}$ & $2,298.4$ & 11.29 \\
\hline \multicolumn{7}{|l|}{ Low variant } \\
\hline $\begin{array}{l}\text { Soma De Silva } \\
\text { (March 1994) }\end{array}$ & $18,711.5$ & -85.8 & -0.46 & $19,721.4^{*}$ & -638.0 & -3.13 \\
\hline W Indralal De Silva (August 1997) & $18,957.7$ & 160.4 & 0.85 & $20,506.2^{*}$ & 146.8 & 0.72 \\
\hline United Nations (1998) & $18,565.0^{*}$ & -232.3 & -1.24 & $20,049.2^{*}$ & -310.2 & -1.52 \\
\hline
\end{tabular}

Sources : De Silva, S. (1994); De Silva, W. I. (1997); UN (1998); Sri Lanka, Department of Census and Statistics (2014)

Percentage Errors in high and low variant projections of group one against 2001 and 2012 observed figures are presented in Table 6.

PEs for Soma De Silva and UN (1998) projections (low variant) are reported negative signs at both 2001 and 2012 censuses. MAPEs for high variant projections against 2001 and 2012 observed are 2.82 and 8.17 per cent respectively. The same indicators for low variant projections are 0.85 and 1.79 per cent. The lowest MAPE values are reported from low variant projections among three variants of group one.

\section{Percentage Error in group two projections against observed 2012}

A population projection which is published in 2007 by W. Indralal De Silva has been taken for the evaluation in group two after the year 2000. This projection has run through a time horizon of 100 years. For the comparison purpose with 2012 observed population, UN (2007) projection has also been taken. The time horizon of the UN (2007) projection is 45 years (2005-2050).

The graphical presentation of population figures of all the variants in both projections against observed 2012 census is shown in Figure 4. For better visible purpose, projected time period is restricted to 50 years from 20012051 in the graphical presentation.

As highlighted in Table 7, the corresponding forecasted figure of 2012 in W. Indralal De Silva's standard variant projection is higher than 2012 observed figure (20.36 vs. 20.68 million). In other words, De Silva has over forecasted the 2012 population by about 300,000 persons. However, the point to emphasise here is that the 2012 census figure refers to 20 March 2012, while De Silva's 2012 figure refers to $1^{\text {st }}$ July 2012 (Mid-year). Therefore, if 2012 census figure of 20.4 is forwarded to the midyear point then the over forecast would disappear significantly - very much similar population figures would be appearing from both sources.

The standard variant projection figure of UN (2007) for 2012 is significantly lower than 2012 observed (20.36 vs. 19.73 million). Further, it is shown that high and low variant projections of UN (2007) is far below compared to De Silva's corresponding high and low variant projection results and the gap is wide across the time span.

Analysis of PEs and MAPEs of all three variants of two projections against 2012 observed is also given in Table 7.

PE values of W. Indralal De Silva's (2007) standard and low projections are always lower than United Nations (2007) projections. For instance, projected standard variant projection results of W. Indralal De Silva (2007) demonstrate only a PE value of 1.59 per cent compared to a value of -3.09 of the UN (2007). Only the high variant of UN (2007) projection takes much closer than De Silva. Due to over and under estimations of values 


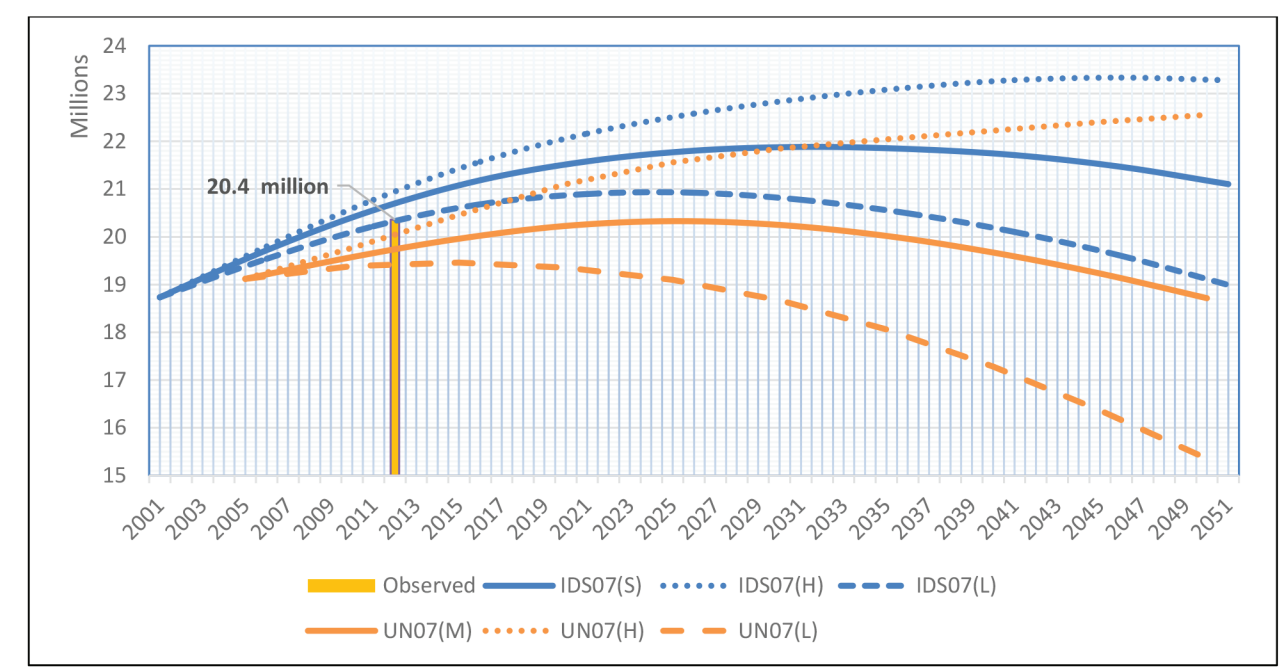

Figure 4: Comparison of projected population figures of 2012 with observed figures (standard, high \& low variants) of group two

Note $\quad$ : IDS07(S), IDS07(H) \& IDS07(L) - W. I. De Silva's 2007 standard, high \& low variants UN07(M), UN07(H) \& UN07(L) - United Nation's 2007 medium, high \& low variants

Sources : De Silva, W. I. (2007); UN (2007); Sri Lanka, Department of Census and Statistics (2014)

Table 7: Percentage error derived by comparing projected population figures of 2012 with observed figures (standard, high \& low variants) of group two

\begin{tabular}{|c|c|c|c|c|}
\hline \multirow{2}{*}{ Projection } & \multicolumn{3}{|c|}{2012 observed $(20,359.4$ persons in ' 000$)$} & \multirow{2}{*}{ MAPE } \\
\hline & Projected ${ }^{*}$ & Actual error & $\mathrm{PE}$ & \\
\hline \multicolumn{5}{|l|}{ Standard variant } \\
\hline W. Indralal De Silva (June 2007) & $20,683.6$ & 324.2 & 1.59 & \multirow{2}{*}{2.34} \\
\hline United Nations (2007) & $19,729.6$ & -629.8 & -3.09 & \\
\hline \multicolumn{5}{|l|}{ High variant } \\
\hline W. Indralal De Silva (June 2007) & $20,933.8$ & 574.4 & 2.82 & \multirow{2}{*}{2.19} \\
\hline United Nations (2007) & $20,043.8$ & -315.6 & -1.55 & \\
\hline \multicolumn{5}{|l|}{ Low variant } \\
\hline W. Indralal De Silva (June 2007) & $20,313.2$ & -46.2 & -0.23 & \multirow{2}{*}{2.44} \\
\hline United Nations (2007) & $19,415.6$ & -943.8 & -4.64 & \\
\hline
\end{tabular}

Note : *values are interpolated

Sources : De Silva, W. I. (2007); UN (2007); Sri Lanka, Department of Census and Statistics (2014)

in 3 variants of both the projections, MAPE values are noted to be significantly high (last column of Table 7).

\section{Age-sex structures in group two projections against 2012 observed}

Age-sex structures in three age groups 0-14, 15-59 and $60+$ years, of 2012 total population in the three variants of each projection and observed in 2012 are plotted in Figure 5.
The observed number of people in the 15-59 age group is the lowest (12.75 million) compared to the corresponding values from all 6 variant projections. In case of age group $60+$ observed has taken the lowest value (2.53 million) too. Observed population of age group 0-14 (5.15 million) in 2012 is the highest among all other projections. Interestingly in the UN projection, only 0-14 population changes among variants but population size of $15-59$ and $60+$ groups remain the same in all three variants (Figure 5). 


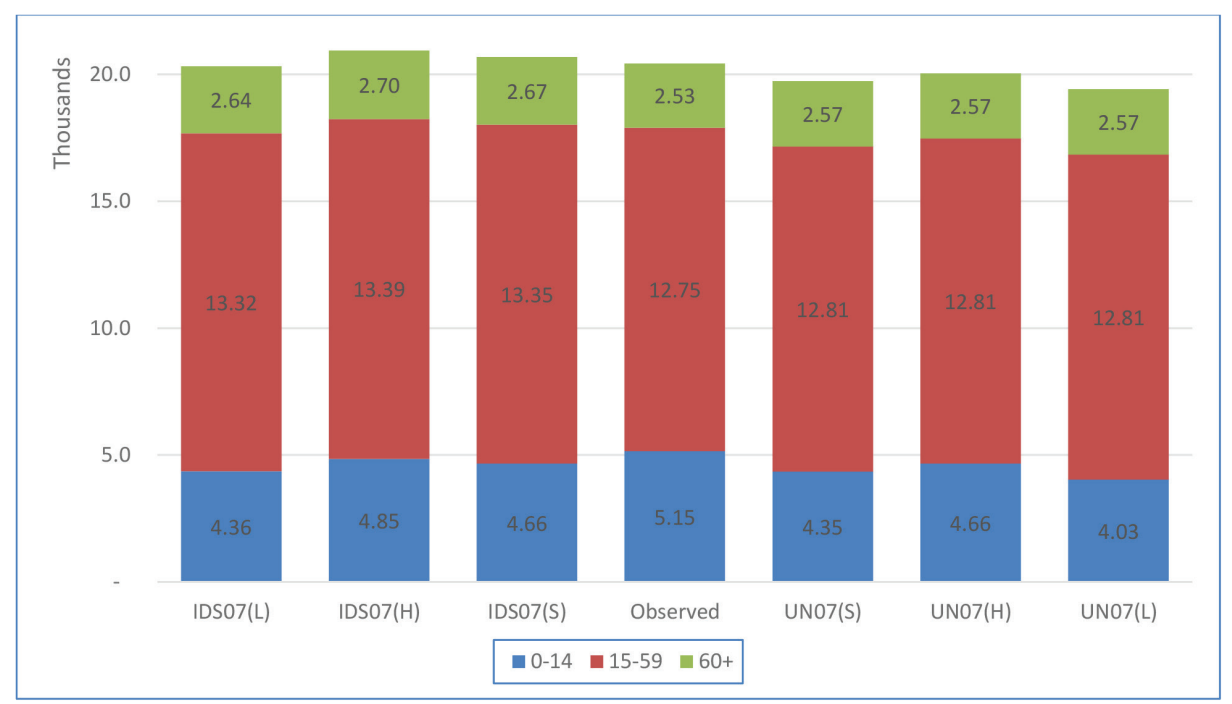

Figure 5: Comparison of total and age structure of projected population of 2012 with observed figures (standard, high \& low variants) of group two

Sources: De Silva, W. I. (2007); UN (2007); Sri Lanka, Department of Census and Statistics (2014)

\section{CONCLUSIONS}

The accuracy of results of existing population projections has been examined in different countries mainly for the common objective of establishing the importance of developing accurate projections. This subject has not been discussed adequately in Sri Lanka primarily due to lack of projection publications for comparison purpose. On the other hand, it may be due to less usage of population projection data in a comprehensive manner in policy formulation, implementation and monitoring. However, the development of accurate population projections is significant as its impact on future forecasting of human necessities, demographic patterns, budgeting and transportation and generally on environmental resource use.

The accuracy of population projection will have a direct impact on the accuracy in many other types of projections, including school going population, labour force, housing etc. For instance, if the child population is over forecasted in the population projection (due to a specific reason), then that would have influenced in over forecasting the school going population. On the other hand, lower forecast of working age population (age 1559 or 15-64 years) in the population projection would lead to lower forecast for the future labour force.

Various estimates of the base year population of 1991 and 1995 have been used by the forecasters primarily due to data quality issue. During the latter part of the $20^{\text {th }}$ century serious lapses in census taking is experienced. Ten years after the 1981 census of population the scheduled census of population of 1991 was not undertaken. After the lapse of 20 years, census of population was carried out in July 2001. However, the 2001 census enumeration was not a complete one - the total population of 2001 was comprised with enumerated population of 18 districts and estimated population of 7 districts. Thus, this issue and the problems related to vital registration, particularly deaths and irregular migration, over the period of 1983-2001, would have contributed to the error in the 2001 base year population (for details refer End Note 1).

Type of errors in projection input data, especially in respect of the base year population seems to be existing in Sri Lanka's past projections. It is evident in differences relating to the same base year population between Soma De Silva's (1994) and W. Indralal De Silva's (1997) projections, and also the difference of same base year between Abeykoon's and UN (1998) projections. These differences of same base year have a definite impact on the projected values in terms of total population, male and female population and age-sex structure.

It can be concluded that projected figures are closer to observed figures in short periods than the long period. In group-one projections, projected total populations of all the variants are much closer to 2001 observed figures than 2012. This variation is clearly explained by corresponding MAPE values. More interestingly, the gaps between projected and observed in 2001 and 2012 for low variant projections are closer than the other 2 
variants. Of the four projections, considered in groupone, UN projection has always deviated from other projections according to the time horizon irrespective of its variants.

In the analysis of group-two projections, although the base year of 2005 of UN (2007) projection is much closer to the observed census year (2012) than De Silva's projection, projected figures of UN are far from observed values. This can be due to inaccuracy of base year population of UN projection. In addition, the gaps between standard to high and standard to low projection of UN are unusually wide along with the time span. In terms of accuracy, De Silva's (2007) standard and low variants are much closer to the observed showing the least error in his low variant projection. Having the highest 0-14 population in 2012 census indicates the occurrence of high number of births than expected in other two projections i.e due to significant fertility increase since the beginning of the new Millennium.

This analysis reconfirms the conclusion made by Stoto (1983) that "the projections are inherently inaccurate". However, quantification of inaccuracy helps to improve population projections. The percentage errors in projections prepared by local forecasters have been found lower according to this study, thereby highlighting the appreciable accuracy of local forecasters.

Further investigations are required in future to help methodically evaluate projections prepared up to 2012 from 1994 in Sri Lanka by local forecasters for improvements, especially in terms of assumptions made by them. Thus, when developing any subsequent projection for Sri Lanka by taking the 2012 census figures as the base population, evaluation of demographic components of fertility, mortality and migration, during the past many decades would facilitate significantly to develop appropriate assumptions for the future.

\section{END NOTES}

1. Sri Lanka has a history of conducting regular population censuses dating as far back as 1871 . However, it is only in the latter part of the 20th century, a significance delay in census taking is experienced. Ten years after the 1981 census of population the scheduled census of population of 1991 was not undertaken, mainly due to civil unrest and its impact on the country's population. The one before the latest census (2012) was carried out on 17 July 2001.
Therefore, by 2001, 20 years will have lapsed without a census of the population of Sri Lanka. During that 20-year period vital registration systems continue to provide statistics, which has been used to make the population estimates. Apart from not having census of population for the past 20 years (1981 to 2000), it is generally believed that after 1983 the quality of the Sri Lankan vital registration system, particularly the registration of deaths and international migration, has deteriorated as pointed out by various researchers such as Fonseka, 1996; Sivarajah et al., 1994 and Sivarajah, 1996 (De Silva, 2008).

Moreover, the 2001 census of population was not a complete enumeration for, the final count was not made in the districts of Jaffna, Mullativu and Killinochchi, and in four other districts namely, Mannar, Vavuniya, Batticaloa and Trincomalee, only a partial enumeration was possible. Although a complete enumeration was made only in 18 out of 25 districts, for the balance 7 districts population was estimated by using information gathered during census pre-enumeration stage.

Thus, the total population of 18.7 million reported for the entire country in the 2001 population census comprises both enumerated and estimated segments (Sri Lanka, Department of Census and Statistics, 2001).

\section{REFERENCES}

Abeykoon A. T. P. L. (1998) Population and manpower resources of Sri Lanka, Colombo: Natural Resources, Energy and Science Authority of Sri Lanka.

De Silva W. I. (1997) Population projection for Sri Lanka: 1991-2041, Colombo: Institute of Policy Studies.

De Silva, S. (1994) Population and labour force projection for Sri Lanka: 1991-2031, Colombo: Department of Census and Statistics.

De Silva, W. I. (2007) A population projection of Sri Lanka for the new millennium 2001-2101: Trends and Implications, Colombo: Institute for Health Policy.

De Silva, W. I. (2008) Construction and Analysis of National and District Life Tables of Sri Lanka 200-2002, Colombo: Social Determinates of Health Initiative, Ministry of Healthcare and Nutrition.

Keilman, N. (1998) How accurate are the United Nations world population projections? Frontiers of population forecasting, Population and Development Review, 24, pp: 15-41.

DOI: https://doi.org/10.2307/2808049 
Khan, H. T. A. and Lutz, W. (2007) How well did past UN population projections anticipate demographic trends in six Asian countries?, Working Paper 507, London: Oxford Institute of Ageing.

DOI: https://doi.org/10.1080/17441730801966964

McDonald, P. (2014) A population projection for Indonesia 2010-2035, Bulletin of Indonesian Economic Studies, 50 (1), pp: $123-129$.

DOI: https://doi.org/10.1080/00074918.2014.896240

Norbert, S. A. (1993) Population growth in Sri Lanka: A trend projection analysis, Sri Lanka Economic Review, 2, pp: 11-22 [Online] Available from : http://dl.nsf.ac.lk/bitstream/ handle/1/13989/ER-18(11)-11.pdf? sequence $=2$ [Accessed: $27^{\text {th }}$ September 2018].

Selvaratnam, S. (1959) Population Projections for Ceylon 1956-1981, Colombo: Planning Secretariat.

Selvaratnam, S., Wright, N. H. and Jones, C. W. (1970) Population Projections for Ceylon 1956-1981, Colombo: Ministry of Planning and Economic Affairs.
Sri Lanka, Department of Census and Statistics (2014) Census of Population and Housing 2012: Key findings, Colombo: Department of Census and Statistics.

Sri Lanka, Department of Census and Statistics (2001) Population by Sex, Age, Religion, Ethnicity According to District and D.S. Divisions, Colombo: Department of Census and Statistics.

Stoto, M. A. (1983) The accuracy of population projections, Journal of the American Statistical Association, 78 (381), pp: 13-20.

DOI: https://doi.org/10.2307/2287094

United Nations (1998) World population prospects- the 1996 revision, New York: United Nations.

United Nations (2007) World population prospects: Sex and age distribution of the world population - the 2006 revision, Vol. II, New York: United Nations.

Wilson, T. (2012) Forecast accuracy and uncertainty of Australian bureau of statistics state and territory population projections, International Journal of Population Research, pp: 20-36.

DOI: https://doi.org/10.1155/2012/419824 